\title{
Berberine ameliorates nonalcoholic fatty liver disease by a global modulation of hepatic mRNA and IncRNA expression profiles
}

\author{
Xinlu Yuan ${ }^{1 \dagger}$, Jie Wang ${ }^{2 \dagger}$, Xiaoyan Tang ${ }^{2}$, Yixue Li ${ }^{2}$, Pu Xia ${ }^{1 *}$ and Xin Gao ${ }^{1 *}$
}

\begin{abstract}
Background: Nonalcoholic fatty liver disease (NAFLD) is a common liver disorder that currently lacks effective treatment. Berberine (BBR), a botanic compound isolated from traditional Chinese medicine, exhibits a potent therapeutic potential for the metabolic disease. The current study aimed to understand the mechanisms underlying the therapeutic effect of BBR in NAFLD.

Methods: We performed systematical analyses on hepatic expression profiles of mRNAs and long noncoding RNAs (IncRNAs) in a high-fat diet (HFD)-induced steatotic animal model with or without BBR treatment. The study was conducted by using the methods of bioinformatics, including hierarchical clustering, gene enrichment and gene co-expression networks analysis. The effect of BBR on the expression profile of some interesting genes was confirmed by quantitative RT-PCR and further studied in a human hepatic cell line, Huh7.
\end{abstract}

Results: We found that a large group of genes including 881 mRNAs and 538 IncRNAs whose expression in the steatotic liver was reversed by BBR treatment, suggesting a global effect of BBR in modulating hepatic gene expression profiles. Among the BBR-regulated genes, we identified several modules and numerous significant genes that were associated with liver metabolism and NAFLD-related functions. Specifically, a conserved IncRNA, MRAK052686, was found strongly correlated with the antioxidant factor Nrf2, and both genes were down-regulated by the steatotic liver. Moreover, the reduced expression of MRAK052686 and Nrf2 was completely reversed by BBR treatment, suggesting a new mechanism accounting for the therapeutic effect of BBR.

Conclusions: The findings for the first time provide a new genetic insight into the pharmaceutical mechanism of BBR in protecting against NAFLD.

Keywords: Berberine, NAFLD, IncRNAs, MRAK052686, Nrf2

\section{Background}

Nonalcoholic fatty liver disease (NAFLD) has become a worldwide health problem that influences approximately $20-30 \%$ of the general population and its prevalence is continuously increasing [1,2]. NAFLD is closely associated with obesity, hyperlipemia and type 2 diabetes, and thus often regarded as atypical hepatic manifestation of the metabolic syndrome [3]. Under certain circumstances, NAFLD can progress from hepatic steatosis to steatohepatitis, leading to an increased susceptibility of

\footnotetext{
* Correspondence: xiapu_fudan@163.com; happy20061208@126.com ${ }^{\dagger}$ Equal contributors

'Department of Endocrinology and Metabolism, Zhongshan Hospital, Fudan University, Shanghai, China

Full list of author information is available at the end of the article
}

cirrhosis and hepatocellular carcinoma [2]. However, there is still a limitation in both mechanistic understanding and clinical management of the disease. Besides life style intervention, several medications, e.g., Metformin, vitamin E and Pioglitazone, are currently applied in the clinic for treatment of NAFLD $[4,5]$. However, these medications often exhibited a limited efficacy and considerable side effects. Therefore, much effort has been focused on the development of new therapeutics for NAFLD.

Berberine (BBR), isolated from the herb Rhizoma Coptidis, has been widely used in traditional Chinese medicine to treat diarrhea and many other inflammatory diseases [6]. Recent studies have revealed a novel therapeutic role of BBR in metabolic disorders, including 
obesity and diabetes $[7,8]$. BBR can function as a cholesterol-lowering drug via a unique mechanism distinct from statins [9]. These findings suggest a potential therapeutic activity of BBR for NAFLD. Indeed, both in vitro and in vivo studies from our previous work and many others have shown that BBR profoundly inhibited lipid synthesis and accumulation in hepatocytes, attenuated hepatic steatosis and hyperlipidemia, and prevented the progression of NAFLD [10-14]. Mechanistically, the therapeutic activity of BBR has been suggested to attribute to its effects of overcoming insulin resistance, reducing endoplasmic reticulum (ER) stress and regulating the signaling pathways, such as the AMPK and JNK pathways [15-18]. More recently, BBR has been shown to modulate gut microbiota, which also account for the therapeutic effect of BBR on the metabolic diseases [19]. It thus appears that multiple mechanisms are involved in the therapeutic effect of $\mathrm{BBR}$, leading us to hypothesize that BBR may have a global effect in modulating gene expression profile in the liver and thereby protecting against hepatic steastosis.

Long noncoding RNAs (lncRNAs) are a novel class of RNA transcripts that are more than $200 \mathrm{bp}$ in length and have little or no protein-coding capacity [20]. According to chromosomal position relative to coding RNAs, lncRNAs are mainly grouped into intergenic, intronic, sense and antisense non-coding RNAs [21]. Most lncRNAs show moderately evolutional conservation and specific transcription [22]. Recently, IncRNAs have been shown widely involved in epigenetic regulation via their direct or indirect interactions with chromatins [23]. Acting as important regulatory molecules, miscellaneous IncRNAs are critically complicated diverse biological processes from normal development and human diseases $[24,25]$. However, there are little studies on lncRNA in NAFLD. In an attempt to further understand the mechanisms underlying the therapeutic effect of BBR, we performed systematical analyses on hepatic expression profiles of mRNAs and lncRNAs in a high-fat diet (HFD)-induced steatotic animal model with or without BBR treatment as reported in the present study.

\section{Materials and methods}

\section{Animals}

Animal studies were approved by the Animal Use and Care Committee of Fudan University and were in conformity with the US Public Health Service Policy on Humane Care and Use of Laboratory Animals. Total 24 healthy male Sprague-Dawley rats weighing around $200 \mathrm{~g}$ were obtained from the Animal Development Center, Chinese Academy of Sciences, Shanghai. All rats were acclimated for 1 week before initiation of the experiment and maintained on a 12/12 h light/dark cycle with free access to food and water. The animals were divided to the following three groups (8 rats per group): (i) Control group (ND group), received a regular rodent chow diet (62.3\% carbohydrate, $12.5 \%$ fat and $24.3 \%$ protein in total calories); (ii)HFD group, fed a HFD (32.6\% carbohydrate, $51.0 \%$ fat and $16.4 \%$ protein) for 24 weeks; and (iii) HFD + BBR group, after 8 weeks of HFD feeding, rats were orally treated with BBR (Sigma, St. Louis, USA) at a dose of $200 \mathrm{mg} / \mathrm{kg} /$ day via a lavage needle and fed on HFD feeding for 16 weeks. Animals received intraperitoneal glucose tolerance tests (IPGTT), weekly measurements of body weight and food intake, and monthly tests of fasting serum insulin and glucose as described previously [12]. Hepatic lipids were extracted according to the method of Folch et al. [26] and triglyceride content was tested as described previously [27]. Fasting serum levels of total cholesterol and low-density lipoprotein cholesterol (LDL-c) were measured using an Amplex Red Cholesterol Assay Kit (A12216, Molecular Probes Invitrogen Detection Technologies) according to the manufacturer's protocol.

\section{Histological examination}

All liver samples were collected after animals were sacrificed in 24th week of the treatment and subsequently fixed in phosphate-buffered $10 \%$ formalin. The right lateral lobule of each liver was embedded in paraffin blocks and stained with hematoxylin and eosin (H\&E) examining the pathologic features of the liver.

\section{Microarrays of mRNAs and IncRNAs}

Arraystar Rat LncRNA microarrays (accession in NCBI Gene Expression Omnibus, http://www.ncbi.nlm.nih.gov/ geo: GPL15690) were used for measuring the expression profiles of lncRNAs and mRNAs in total 15 rats (i.e., 3 groups at 5 replicates). The sample preparations and microarray hybridization were performed based on the manufacturer's protocols. Briefly, $1 \mu \mathrm{g}$ of total RNA from each sample was amplified and transcribed into fluorescent cRNA using Agilent's Quick Amp Labeling kits according to the manufacturer's protocol (version 5.7, Agilent Technologies). The labeled cRNAs were hybridized onto the Rat LncRNA Arrays $(4 \times 44 \mathrm{~K}$, Arraystar, Rockville, USA). After washing, the arrays were scanned by the Agilent Scanner G2505B. Raw signal intensities were normalized in quantile method by GeneSpring GX v11.5.1. After filtration of low intensity RNAs, those RNAs with flags in Present or Marginal at least 1 out of 15 samples were chosen for further analysis. Differentially expressed genes were identified by using the Limma package [28]. The threshold set for significantly differential genes was an absolute log value of fold change $\geq 0.6$ and a $p$ value $<0.05$. 


\section{Hierarchical clustering and gene enrichment analysis}

We took Euclidean distance with a complete linkage to hierarchically cluster the genes across the samples. Unsupervised hierarchical clustering and the visualization of heatmap were performed using the $\mathrm{R}$ platform (http://www.r-project.org/). Gene function enrichment analysis was performed by using DAVID [29]. The significance of enrichment was calculated by hypergeometric test and adjusted by Benjamini method. GO (gene ontology) and KEGG (kyoto encyclopedia of genes and genomes) were accessed from the databases of http://www.geneontology.org/ and http://www.genome.jp/, respectively [30,31].

\section{Gene module-trait relationships}

We analyzed associations between gene modules and the traits by using WGCNA (weighted correlation network analysis) package [32]. The package assessed module-trait relationships by correlating of each module eigengene (the first principal component of gene expression in the module) with the traits.

\section{Construction of gene co-expression networks}

We constructed and analyzed the co-expression network of lncRNAs and mRNAs using WGCNA package. The weight of edges was scaled by a soft power of correlation coefficient of parewise genes, and the soft power was set to 16 after stimulation. The visualization and layout of network were achieved by cytoscape software [33].

\section{Prediction for protein-coding potential and interaction propensity of IncRNAs}

The protein-coding potential of lncRNAs was predicted by PhyloCSF which calculates codon substitution frequencies (CSF) determining whether a multispecies nucleotide sequence is conserved protein-coding region [34]. The more positive value of CSF indicates a higher potential to code protein, vice versa. Multiple alignments of 8 vertebrate genomes are downloaded from UCSC (http://genome.ucsc. edu/). We used catRAPID to predict the interaction propensity of lncRNAs with proteins [35]. The software catRAPID computes interaction propensity (IP, a measure based on physico-chemical profiles of ribonucleoprotein complexes) and discriminative power (DP) for assessing the potential of interaction. DP above $75 \%$ represents highly confident IP.

\section{Identification of human homologous of the IncRNA MRAK052686}

According to genome-wide alignment from UCSC (http:// genome.ucsc.edu) and chromosomal position, we found that human MRAK052686 is located around ZBTB20. A direct searching of MRAK052686 in human genome indicated the same chromosomal location as mentioned above. By searching this human locus in ENSMBL database (http://www.ensembl.org) and then aligning sequences, we found 6 of 11 non-coding transcripts with a high similarity to rat MRAK052686 (Additional file 1: Table S1 and Additional file 2: Figure S1). These six lncRNAs were thus regarded as human homologues of MRAK052686.

\section{Cell culture, treatment and intracellular lipid staining}

Huh7, a human hepatoma cell line, was obtained from American Type Culture Collection (Manassas, VA) and routinely cultured in DMEM supplemented with 10\% fetal bovine serum. For treatment, a stock solution of $100 \mathrm{mM}$ oleic acid with $11 \%(\mathrm{w} / \mathrm{v})$ fatty acid-free BSA was prepared. When reaching a $70 \%$ confluence, cells were washed with PBS and exposed to $100 \mu \mathrm{M}$ OA for $24 \mathrm{~h}$ in the presence or absence of $10 \mu \mathrm{M}$ BBR. For intracellular lipid staining, cells were washed with PBS three times and fixed with $4 \%$ paraformaldehide for 30 min. Intracellular lipids were stained with Nile Red (Sigma, St. Louis, USA) for $15 \mathrm{~min}$ and nuclei stained with DAPI for an additional $5 \mathrm{~min}$. Images were photographed with an inverted fluorescence microscope (Leica DM2000, Wetzlar, Germany).

\section{Real-time quantitative PCR}

All rats were euthanized and their livers were collected after 24 weeks treatment. Total RNA was isolated from liver tissues using Trizol reagent (Invitrogen, Carlsbad, CA). cDNA was synthesized by reverse transcription in a $20 \mu \mathrm{L}$ reaction system using PrimeScript kit (Takara, Tokyo, Japan). The reverse transcription reactions were conducted at $37^{\circ} \mathrm{C}$ for $15 \mathrm{~min}$ and inactivated at $85^{\circ} \mathrm{C}$ for 30 s using an automatic thermocycler according to manufacturer's instruction. Real-time quantitative PCR (RT-qPCR) was performed with an Applied Biosystems 7500 using SYBR Premix EX Taq (Takara, Tokyo, Japan). A total $10 \mu \mathrm{L}$ reaction mixture contained of $5 \mathrm{ng}$ sample cDNA, $5 \mu \mathrm{M}$ specific forward and reverse primers, and $5 \mu \mathrm{L}$ SYBR Premix EX Taq. The sequences of primers used for RT-qPCR are shown in Additional file 1: Table S2. The PCR reaction was consisted of an initial denaturation cycle at $95^{\circ} \mathrm{C}$ for $10 \mathrm{~min}$ and 40 amplification cycles of $95^{\circ} \mathrm{C}$ for $15 \mathrm{~s}$ and $60^{\circ} \mathrm{C}$ for $1 \mathrm{~min}$. The relative gene expression was calculated using the $2^{-\Delta \Delta C t}$ method [27]. The same reaction was carried out in triplicate with $\beta$-actin as an internal control. Experiments of each condition were conducted in 3 replicates.

\section{Statistical analysis}

All data were presented as mean \pm SEM. Significance was assessed by one-way ANOVA followed by Tukey's test and LSD for multiple comparisons. Chi-square test was used to assess the significance of variables in the contingency table. All statistics were performed in $\mathrm{R}$ 
platform. A value of $\mathrm{p}<0.05$ was considered statistically significant.

\section{Results}

\section{BBR ameliorates hepatic steatosis in HFD-fed rats}

BBR has been reported to prevent the development of NAFLD in animal models [12,36,37]. To confirm the therapeutic effect of BBR, we examined the effect of BBR on the liver in HFD-induced obese rats. As shown in Figure 1, HFD-fed animals exhibited moderate to severe hepatic steatosis with typical histological features of cell swelling and large amount of fat accumulations within hepatocytes. Remarkably, HFD-induced steatosis was evidently ameliorated by BBR treatment, as reflected in a significant reduction of ballooning cells and Mallory bodies (Figure 1A). Moreover, BBR treatment significantly lowered hepatic TG contents and serum levels of TC and LDL-c by $13.7 \%, 28.1 \%$ and $41.9 \%$, respectively, in comparison with the HFD-fed animals (all $\mathrm{p}<0.05$, Figures $1 \mathrm{~B}$ and $1 \mathrm{C}$ ).

\section{BBR systematically modulates hepatic gene expression} In an attempt to understand the molecular basis of the efficacy of BBR, we performed a microarray-based analysis of hepatic gene expression profiles in ND, HFD and HFD + BBR treated animals. After normalization and filtration of microarray data, total 14, 319 mRNAs and 9,531 lncRNAs were identified for further analysis.
By comparison of HFD + BBR with HFD group, we identified 531 and 407 differentially expressed mRNAs and IncRNAs, respectively (Figure 2A). In addition, the second comparison of HFD with ND group revealed that 4, 612 mRNAs (including 2, 719 up-regulated and 1, 893 down-regulated mRNAs) and 2, 813 lncRNAs (consisting of 818 up-regulated and 1, 995 downregulated lncRNAs) were differentially expressed, of which 362 mRNAs and 284 lncRNAs were overlapped with the first comparison (Figure 2A). Among the overlapped and reversely expressed genes in two comparisons, BBR significantly up-regulated more lncRNAs (241 lncRNAs vs. 121 mRNAs) and down-regulated more mRNAs (233 mRNAs vs. 37 lncRNAs) (Figure $2 \mathrm{~A}, \mathrm{p}<1.0 \mathrm{E}-10$, chi-squared test). Moreover, among the 7,425 genes whose expression was altered in HFD group, 6,344 (85.4\%) genes including 2,399 lncRNAs were reversed towards a normal expression profile after BBR treatment (Figure 2B, $\mathrm{p}<1.0 \mathrm{E}-10$ ). In addition, by comparing HFD and HFD + BBR with ND group, respectively, we found much fewer differences in mRNA and IncRNA expression between HFD + BBR and ND than that between HFD and ND groups (476 mRNAs and 374 lncRNAs versus 2131 mRNAs and 1402 lncRNAs, Additional file 3: Figure S2). Taken together, the data suggest a potent effect of BBR on a global regulation of hepatic gene expression in the steatotic liver trending towards a normal profile, including abundant lncRNAs.

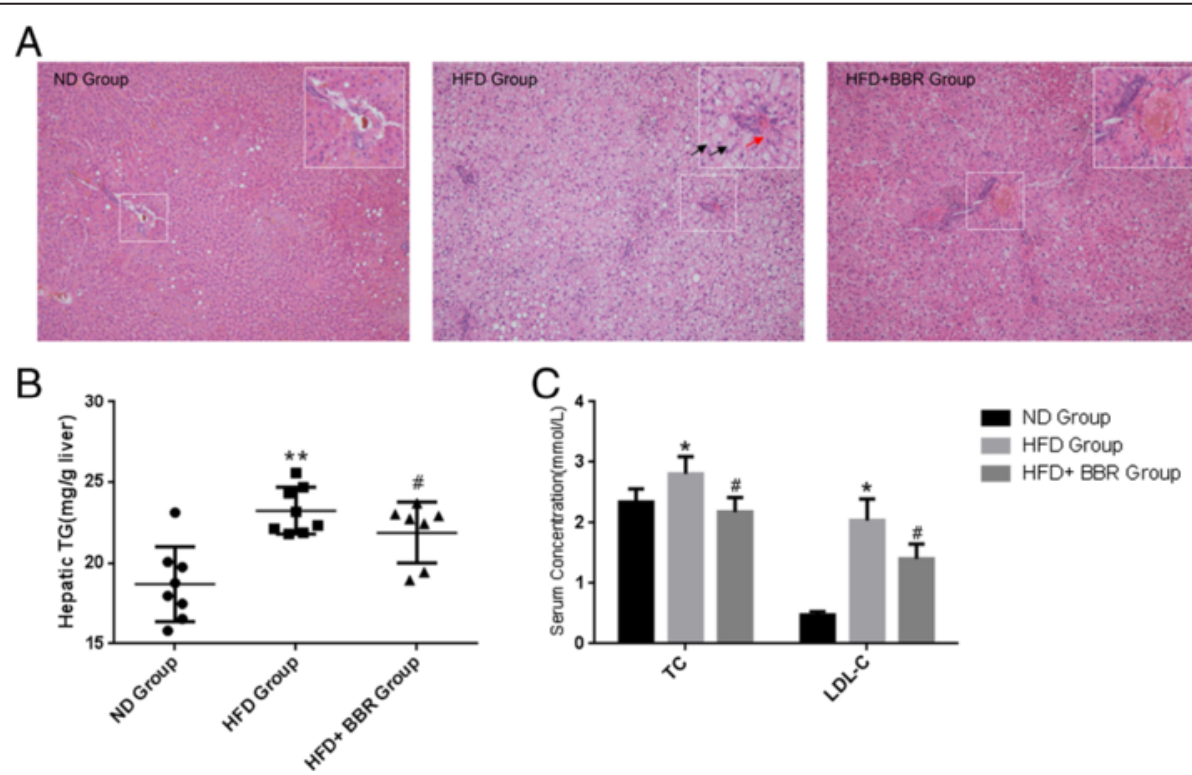

Figure 1 BBR ameliorates hepatic steatosis in HFD-fed rats. (A)The livers isolated from rats fed with a normal diet (ND), high-fat diet (HFD) or HFD plus Berberine (HFD + BBR)were stained with hematoxylin and eosin. Photographs are at $200 \times$ magnification. A large magnification is shown in the white box. Ballooning cells and Mallory bodiesare shown in black and red arrows, respectively. (B) Hepatic TG contents, and (C) serum TC and LDL-c levels were measured in the experimental animals as described in "Methods". Data are mean \pm SEM. * $p<0.05,{ }^{* *} p<0.01$ versus ND; \# $p<0.05$ versus HFD (assessed by one-way ANOVA followed by Tukey's multiple comparison tests). BBR, berberine; HFD, high-fat diet; ND, normal diet; TG, triglyceride; TC, cholesterol. 

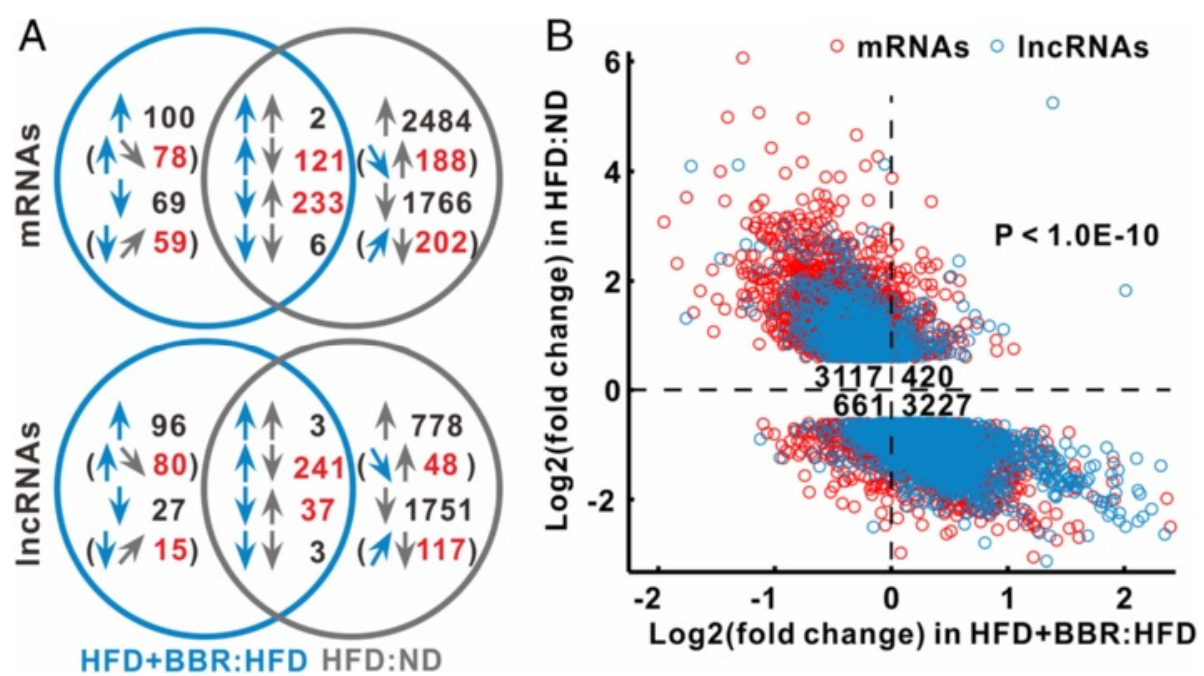

Figure 2 BBR systematically regulates hepatic gene expression. (A) A Venn diagram of differentially expressed genes in the experimental rats. The cyan and grey arrows represent the comparisons of HFD + BBR with HFD group (HFD + BBR:HFD) and HFD with ND group (HFD:ND), respectively. The upward arrows indicate up-regulation and downward, down-regulation. Sloped arrows in brackets indicate genes whose expression is not significantly altered but meets our criteria of BBR-regulated genes. Numbers in brackets indicate a subset of the number immediately above. The red number is the count of BBR-regulated genes. (B) BBR reversed most differentially expressed genes in HFD relative to ND group. The numbers in the center indicate the count of genes in corresponding quadrant. The $\mathrm{p}$ value was calculated with the Chi-squared test.

\section{Expression profiles of BBR-regulated genes}

In order to further characterize the effect of BBR on hepatic gene regulation, we specifically grouped the genes whose expression was potentially regulated by BBR. To this end, we used the following criteria to determine the genes as BBR-regulated genes: (i) the gene expression was inversely changed in two comparisons, and (ii) absolute $\log$ value of fold change $\geq 0.6$ in the first comparison between HFD + BBR and HFD groups. Accordingly, total 881 mRNAs and 538 lncRNAs were identified as BBR-regulated genes whose expression patterns were reversed after BBR treatment in comparison to HFD group (Figure 2A, Additional file 4: Table S3).

For assessing the association of lncRNAs with mRNAs, we performed hierarchical clustering of BBR-regulated genes across the three experimental groups. HFD + BBR group exhibited a similar expression profile of BBR-regulated genes to the ND group (Figure 3A). Furthermore, we divided
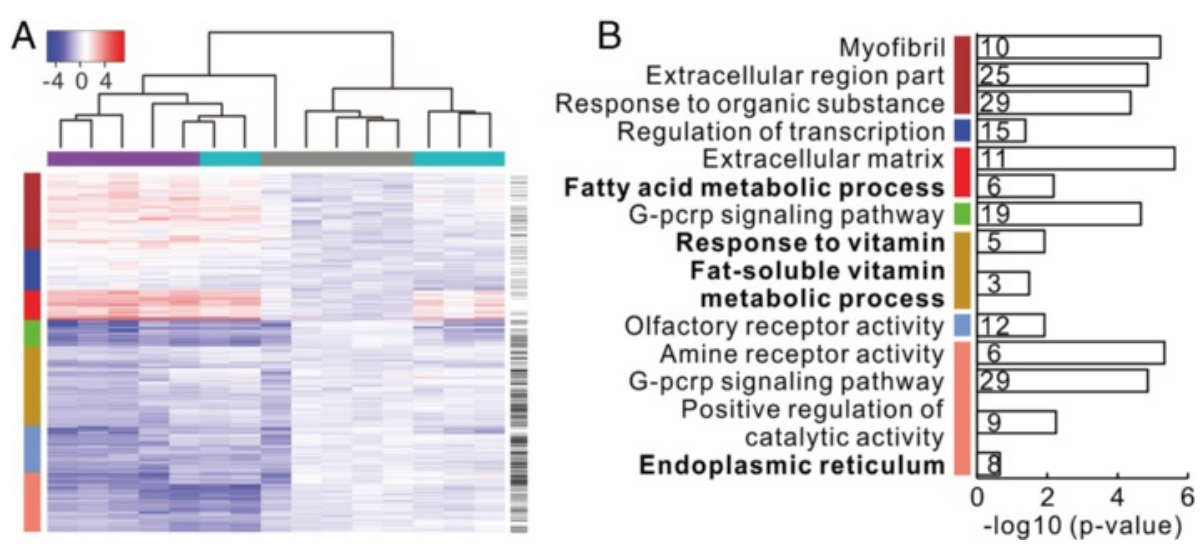

Figure 3 Hierarchical clustering and functions enrichment analyses of BBR-regulated genes. (A) Each column represents samples from ND (grey), HFD (purple) or HFD + BBR (cyan) group. Each row indicates genes, in which IncRNAs are labeled with black bars on the most right column. Each cell of the heat-map reflects expression value above or below the pool of control rats fed ND. The legend of expression value is shown on the upper left. Based on gene clustering tree (Additional file 5: Figure S3), BBR-regulated genes are divided into seven modules, indicating with the most left color column: brown, blue, red, green, dark golden, light blue and salmon. (B) Representative enriched functions for seven BBR-regulated modules. The number on the right of vertical axis indicates the count of enriched genes in corresponding function. The NAFLD-related functions highlight in bold. The color bars on the left of vertical axis represent the modules. More details of the enriched functions for each module are shown in Additional file 5: Table S3. 
BBR-regulated genes into seven modules (termed as BBR-regulated modules) based on gene clustering trees (Additional file 5: Figure S3). These modules represent distinct expression patterns. In these modules, lncRNAs were not uniformly distributed, whereas lncRNAs were evidently enriched in the dark golden and light blue modules (Figure 3A).

Based on the above seven BBR-regulated modules, we assessed the correlation of NAFLD-related traits with the module eigengenes using WGCNA package [32]. We found that salmon module was mostly correlated with triglyceride metabolism and red module associated with lipoproteins (Additional file 6: Figure S4). Of note, function enrichment analysis by using DAVID [29] indicated that the salmon and red modules are likely associated with NAFLD, including the genes involved in endoplasmic reticulum (ER) function and fatty acid metabolism (Figure 3B, and Additional file 1: Table S4). In addition, mRNAs in dark golden module is also related to NAFLD, including the genes involved in fat-soluble vitamin metabolic process. Collectively, these data indicate that a large number of BBR-regulated genes including lncRNAs are indeed functionally associated with NAFLD (especially those from salmon, red and dark golden modules).

\section{The InCRNA MRAK052686 is a significant BBR-regulated gene} Given the numerous BBR-regulated genes as identified above, we assessed gene significance by analyzing association of gene expression with traits. The genes with a high significance for trait and strong correlation in module were characterized as potentially important genes. Accordingly, we identified two significant IncRNAs, MRAK052212 and MRAK080926, from the salmon module that was highly correlated with triglyceride metabolism (Additional file 7: Figure S5A). The function annotation of associated mRNAs indicated that MRAK052212 and MRAK080926 may participate in lipid metabolism and ER to Golgi vesicle-mediated transport process (Additional file 1: Table S5). Furthermore, we identified another two most significant proteincoding genes from the red module, including $L y z 2$ and Tnfrsf 18, which were highly correlated with LDL trait (Additional file 7: Figure S5B). Lyz2 is suggested to be associated with catabolic process in ER according to gene ontology (GO). Then, we analyzed co-expression subnetworks from the significant genes and their first neighbor genes in each NAFLD-related module. In the sub-networks derived from salmon and red modules, we identified three potentially important lncRNAs, including AF034247, MRuc007jok and BC166462, which were all connected with enriched protein-coding genes (Additional file 8: Figure S6). In the dark golden module, a sub-network was delineated based on two enriched and strong coexpressed genes, Eif2ak2 (also known as Prkr) and Adh1 (Pearson's correlation coefficient, $\mathrm{R}=0.59, \mathrm{p}=0.02$ ). Interestingly, in this sub-network we found an IncRNA, MRAK052686 that was strongly associated with Eif2ak2 $(\mathrm{R}=0.59, \mathrm{p}=0.022)$ and Nrf2 (also known as $\mathrm{Nfe} 2 \mathrm{l} 2, \mathrm{R}=0.91, \mathrm{p}=2.7 \mathrm{e}-06$ ) (Figure 4). Both Eif2ak2 and Nrf2 have been previously documented being associated with the pathogenesis and progression of NAFLD [38]. In addition, according to the KEGG Pathway Database, Nrf2 was a direct downstream target of Eif2ak2 in ER stress response pathways. Furthermore, the IncRNA MRAK052686 was co-expressed with several other NAFLD-related genes, including the fatty acid binding protein $F a b p 7$ and Gcs1, which are involved in ER protein processing

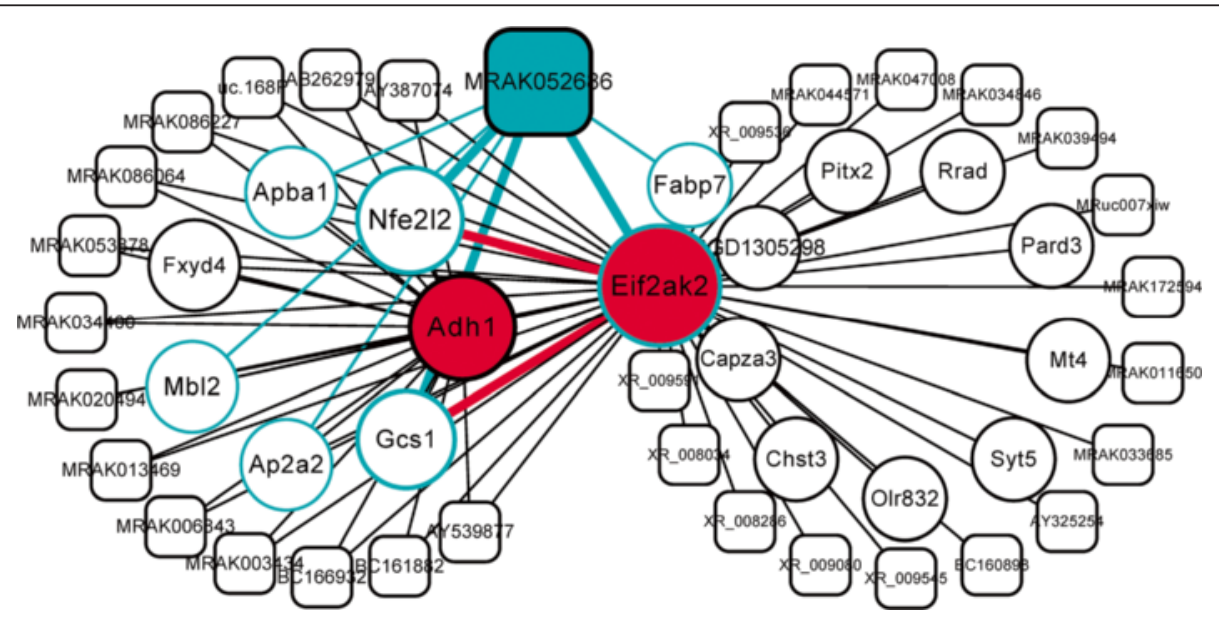

Figure 4 Co-expression sub-networks derived from enriched genes in dark golden module. Nodes of sub-networks consist of enriched genes and their first neighboring genes. The threshold for edges of co-expression network is 0.25 for weighted correlation. The red genes, Eif2ak2 and Adh1, were identified by enrichment analysis showing in Figure 3B. The potentially important IncRNA MRAK052686 and its associated mRNAs are highlighted in cyan color. Rectangles and circles represent IncRNAs and mRNAs, respectively. 
(Additional file 1: Table S5). Together, these results suggest that the lncRNA MRAK052686 may play important roles in NAFLD by affecting the genes that regulate cellular stress response and protein processing in ER. Besides MRAK052686, we found more lncRNAs (accounting for $65 \%$ ) connected to the NAFLD-related genes in sub-networks of dark golden and salmon modules (Figure 4 and Additional file 8: Figure S6), further indicating an important role of lncRNAs in NAFLD.

MRAK052686 is located in 3'UTR of protein-coding gene $Z b t b 20$, which is an important regulator of glucose homeostasis and has been reported to associate with liver dysfunction [39]. However, we found no difference in Zbtb20 expression between HFD + BBR and HFD groups (data not shown) and no correlation of $Z b t b 20$ to MRAK052686. This suggests that MRAK052686 is a transcript independent of Zbtb20. Further analysis indicated that codon substitution frequency of MRAK052686 is strongly negative (Additional file 9: Figure S7). Moreover, we found no protein sequence significantly matched with MRAK052686 from NCBI reference protein database (data not shown), further suggesting that MRAK052686 has no or little potential to code protein.

\section{BBR restores the decreased expression of MRAK052686 and Nrf2 in steatotic liver}

Having identified the lncRNA MRAK052686 and the related gene Nrf2 from the NAFLD-related modules, we wanted to clarify whether their expression was correlated in NAFLD and whether regulated by BBR treatment. By conducting RT-qPCR assays, we confirmed that both MRAK052686 and Nrf2 expression was significantly down-regulated in the livers of HFD group compared with the controls $(\mathrm{p}<0.01$, Figure $5 \mathrm{~A})$. Moreover, the reduced expression of MRAK052686 and Nrf2 was markedly reversed by BBR treatment $(\mathrm{p}<0.05$, Figure $5 \mathrm{~A})$. In addition, a significant positive correlation between hepatic expression levels of MRAK052686 and Nrf2 existed across ND, HFD and HFD + BBR groups $(\mathrm{R}=0.76$, $\mathrm{p}=0.00015$, Figure $5 \mathrm{~B})$, further supporting a strong co-expression profile of these two genes in the liver.

By searching MRAK052686 homolog in human genes, we found 6 homologous variants from ENSMBL database (Additional file 1: Table S1 and Additional file 2: Figure S1). Among 6 variants, four of them (i.e., ZBTB20-008, -013, -016 and -017) were detected in human Huh7 hepatocytes (Figure 6). To further clarify whether the expression of these noncoding transcripts is also associated with hepatic steatosis in human, we treated Huh7 cells with oleic acid (OA) [26], which is regarded as an established steatotic cell model. As shown in Figure 6, cells exposed to $100 \mu \mathrm{M}$ OA for $24 \mathrm{~h}$ resulted in an evident lipid accumulation, whereas BBR treatment significantly prevented the OA-induced steatosis (Figure 6A). In keeping with the findings from the HFD-fed animals, all the detectable variants of noncoding ZBTB20 transcripts were significantly down-regulated in OA-treated hepatocytes, which were.completely reversed by BBR
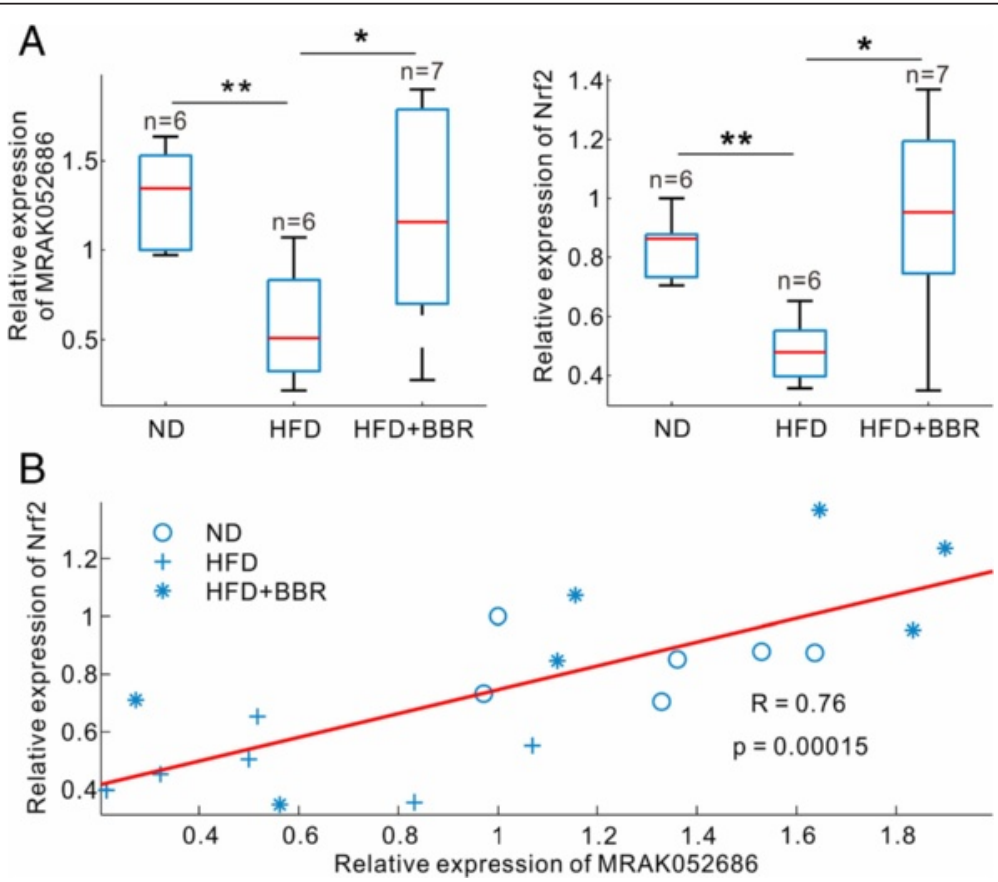

Figure 5 Co-expression of MRAK052686 and Nrf2 across three groups of the rats. (A) Levels of MRAK052686 and Nrf2 expression in livers were measured by RT-qPCR and normalized by $\beta$-actin expression. Significance was assessed by one-way ANOVA followed by Tukey's multiple comparison tests. Data are shown as mean \pm SEM $(n \geq 4)$. Pearson's correlation coefficient $(R)$ and corresponding $p$ value of the fitted line are shown in (B). 


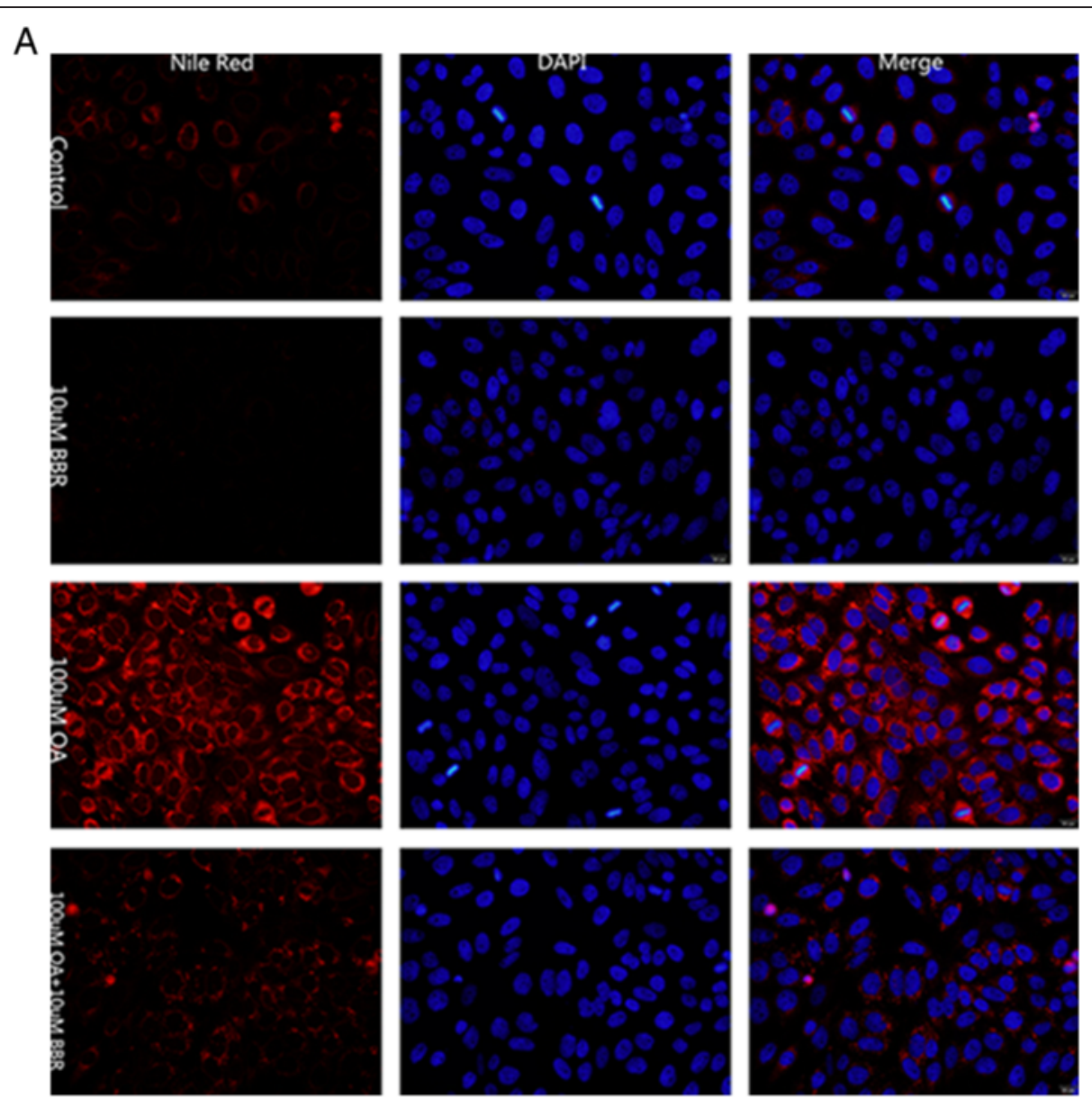

B

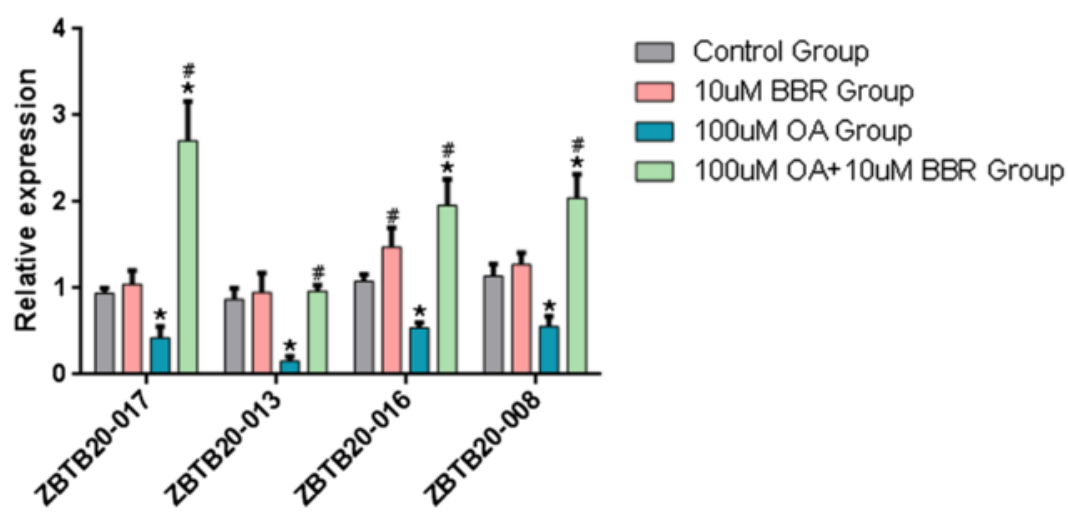

Figure 6 The expression pattern of noncoding ZBTB20 transcripts in human Huh7 cells. (A) Huh7 cells were treated for $24 \mathrm{~h}$ with OA in the presence or absence of BBR $(10 \mu \mathrm{M})$, and then stained with Nile Red. Photographs were taken by a fluorescence microscopy and shown at $400 \times$ magnification. (B) Four human homologues of MRAK052686 were measured by RT-qPCR in Huh7 cells treated with BBR, OA or BBR + OA as indicated $(n=3)$. Data are shown as mean \pm SEM. ${ }^{*} p<0.05$, vs control and ${ }^{\#} p<0.05$, vs OA alone. Significance was assessed by one-way ANOVA followed by Tukey's multiple comparison tests. OA, oleic acid; BBR, berberine.

treatment (Figure 6B). Taken together, the findings not only indicate the connection of noncoding ZBTB2O transcripts with hepatic steatosis, but also suggest that the protective effect of BBR against NAFLD is associated with the expression of these lncRNAs.

\section{Discussion}

Multiple lines of evidences from both in vivo and in vitro studies have demonstrated a therapeutic potential of the botanic compound BBR in metabolic diseases, including obesity and diabetes $[7,8,10-14]$. In the present, we were 
able to confirm the effect of BBR in amelioration of NAFLD in HFD-induced obese rats as reported previously $[12,14]$. By analyzing gene expression profiles, we identified 5, 271 mRNAs and 3, 265 lncRNAs, which displayed differential expression patterns among the control, HFD and HFD + BBR treated animals. We further refined a large group of BBR-regulated genes, including $881 \mathrm{mRNAs}$ and 538 lncRNAs whose expression was reversed between HFD and HFD + BBR groups, demonstrating a potent effect of BBR in globally altering hepatic gene expression. Moreover, by modularization of the BBR-regulated genes, we delineated several modules and identified numerous significant genes exhibiting a wide association with liver metabolism and NAFLD-related functions. The findings thus, for the first time, provide a genetic basis for understanding the pharmaceutical mechanism of BBR in protection against multiple metabolic diseases, including NAFLD.

Of interesting, not only were over 800 of BBR-regulated mRNAs identified in the current study, but also more than 500 aberrant lncRNAs in NAFLD were reversed by BBR treatment (Figure 2A). The number of altered lncRNAs was comparable with that of mRNAs. Although the precise roles of these lncRNAs in NAFLD are currently unknown, lncRNAs have emerged as important regulators of gene expression related to metabolic homeostasis or dysfunction. For instance, it was reported that near two hundreds of lncRNAs were regulated during the process of adipogenesis and many of them were bound at their promoters by key transcription factors such as PPAR $\gamma$ and CEBP $\alpha$ [40]. Furthermore, RNAi-mediated loss of function screens identified numerous lncRNAs that were required for proper adipogenesis [41]. There is a large body of evidences that lncRNAs can regulate gene expression both in cis and in trans by forming RNA-protein, DNA-RNA, protein-DNA and RNA-RNA interactions [41]. In the current study, we found that a total of $538 \operatorname{lncRNAs}$ were regulated in the liver by BBR treatment in HFD-induced obese rats. Among these BBR-regulated lncRNAs, a set of IncRNAs was significantly associated with triglyceride trait (Additional file 8: Figure S6). In the analysis of co-expression sub-networks, we identified numerous lncRNAs were located in the salmon and dark golden modules, which are functionally linked with NAFLD-related genes (Figure 4 and Additional file 8: Figure S6). A significant example of these lncRNAs is MRAK052686. We found that MRAK052686 expression associated with Eif2ak2 and Nrf2 (Figure 4), the two genes that are critically involved in NAFLD. By bioinformatics prediction, we found the interaction propensity and discriminative power of $\mathrm{Nrf2}$ and Eif2ak2 interacting with MRAK052686 are far stronger than that of the control genes Actb and Gapdh (Additional file 10: Figure S8). Indeed, a strong co-expression of MRAK052686 and Nrf2 was demonstrated by either microarray analysis or the experimental data from HFD-fed animals (Figure 5). This implies a potential role of MRAK052686 in regulation of $N r f 2$ expression.

Nrf2 is a master transcription factor that regulates expression of many genes that are involved in antioxidant, metabolism and ER stress response [38,42]. In mammalian, Nrf2 is regarded as a major cellular sensor of oxidative stress, which constitutes downstream the PERK (PKR-like ER-kinase) pathway during ER stress response [41]. Upon PERK activation, Nrf2 is activated and binds to the antioxidant response elements in the regulatory regions of target genes, thereby regulating ER stress response [43,44]. Besides, Eif2ak2, another associated gene with MRAK052686, is actually a homologue of PERK. Moreover, Gcs1 that was also co-expressed with MRAK052686 functions in ER protein processing and regulates ER stress response. Indeed, ER stress has been well documented as a pivotal pathogenic event contributing to the process of hepatic steatosis $[45,46]$. The findings that MRAK052686 associates with several key molecules of the ER stress response pathways suggest that MRAK052686 may play important roles in NAFLD by affecting ER stress response, which warrants further investigations.

In the present study, another key finding is that most of the aberrantly expressed genes, including numerous lncRNAs, in HFD-induced hepatic steatosis were reversed by BBR treatment. In modularization of the sub-networks from salmon and dark golden modules, much more lncRNAs were co-expressed with enriched NAFLD-related mRNAs. This not only demonstrates a global effect of BBR on the alteration of gene profiles, but also suggests a population of lncRNAs as a whole target of the therapeutic action of BBR. Correspondingly, BBR exerts its effect to alleviate NAFLD through a systematic modulation of gene expression including lncRNAs in the liver, providing a potential novel therapeutic agent for the treatment of NAFLD.

\section{Conclusions}

By analyzing hepatic gene expression profiles, we found that HFD-induced steatosis in rats resulted in a globe alteration in hepatic gene expression, which was significantly reversed by BBR treatment. Several modules of BBR-regulated genes, including abundant lncRNAs, were identified by bioinformatics analysis. Among these BBR-regulated genes, we found that the IncRNA MRAK052686 and its associated gene Nrf2 appear implicating in the pathogenesis of NAFLD. Thus, the study provides a new genetic insight into the pharmaceutical mechanism of BBR in protecting against NAFLD. 


\section{Additional files}

Additional file 1: Table S1. Sequence similarity of transcript variants of human homologous with rat MRAK052686. Table S2. The primers of genes for RT-qPCR. Table S4. Detailed function annotation of genes in seven modules. Table S5. Function annotation of mRNAs associated with MRAK052686.

Additional file 2: Figure S1. Human chromosomal locations of MRAK052686 and its homologs. The conserved regions between MRAK052686 and its homologs were connected with dash lines. The thick and thin bars in gene models indicate protein-coding and non-protein coding regions, respectively.

Additional file 3: Figure S2. A Venn diagram illustrates the number of genes that were differentially expressed in comparisons of HFDor BBR-treated with ND group. The cyan arrows represent the comparison of HFD+BBR with ND group (HFD+BBR:ND) and grey arrows for the HFD:ND comparison. The upward and downward arrows indicate up-regulated and down-regulated expression, respectively.

Additional file 4: Table S3. The list of BBR-regulated genes. Additional file 5: Figure S3. Hierarchical clustering tree of BBRregulated genes. Each row represents the rat sample from ND (grey), HFD (purple) or HFD+BBR (cyan) group. The columns indicate BBRregulated genes, which are divided into seven modules (the top color column from right to left: brown, blue, red, green, dark golden, light blue and salmon). Each cell of the heat-map reflects expression value above or below pool control level. The legend of expression value is shown on the upper left.

Additional file 6: Figure S4. Relationship of gene modules with rat traits. Each row corresponds to a module eigengene, column to a trait. Each cell contains the corresponding correlation ( $p$ value, calculated by WGCNA package) and is colored by correlation according to the color legend on the right. $\mathrm{CHOL}$, cholesterol; $\mathrm{TG}$, triglyceride; $\mathrm{HDL}$, high density lipoprotein; LDL, low density lipoprotein. The left color bars represent the modules consistent with Figure 3.

Additional file 7: Figure S5. Gene significance of traits in salmon and red modules. (A) Gene significance of triglyceride in salmon modules. (B) Gene significance of LDL (low density lipoprotein) in red modules. The definition and calculation of gene significance and module correlation see Methods part. Circle and star points represent mRNAs and IncRNAs, respectively. The dash lines on the upper right are threshold sets for most significant genes labeled by larger markers and gene names. The linear fit curve with Pearson correlation (R) and significance ( $p$ value) of scatter points are shown.

Additional file 8: Figure S6. Co-expression sub-network derived from enriched genes in salmon and red modules. Nodes of sub-network consist of enriched genes and their first neighboring genes. The threshold of co-expression network is 0.25 for weighted correlation. The red genes were identified by enrichment analysis showing in Figure 4. The potentially important IncRNAs are labeled in cyan color. Rectangles and circles represent IncRNAs and mRNAs, respectively.

Additional file 9: Figure S7. Location and coding potential of MRAK052686. MRAK052686 (blue) are transcribed from sense strand of protein-coding gene Zbtb20 (green). The thick and thin bars of Zbtb20 represent protein-coding and untranslated regions, respectively. The higher codon substitution frequency corresponds to stronger protein-coding potential.

Additional file 10: Figure S8. The heat-map indicating an interaction of MRAK052686 with Nrf2 and Eif2ak2. The vertical index on the left of each heat-map is position of corresponding protein. IP and DP are abbreviations of interaction propensity and discriminative power, respectively. Higher IP and DP indicate more potential interaction.

\section{Competing interests}

The authors declare that they have no competing interests.

\section{Authors' contributions}

$\mathrm{XY}$ and JW carried out the majority of experiments, participated in data analysis and drafted the manuscript. XT and YL participated in the bioinformatics analysis. XG and XP conceived of the study, participated in its design and coordination and helped to draft the manuscript. All authors read and approved the final manuscript.

\section{Acknowledgements}

This work was supported by grants from the Major State Basic Research Development Program of China (2012CB524906 to X.G.; http://www.973.gov. cn/Default_3.aspx), National Natural Science Foundation of China (81270933 to X.G. and 81370937 to P.X.).

\section{Author details}

'Department of Endocrinology and Metabolism, Zhongshan Hospital, Fudan University, Shanghai, China. ${ }^{2}$ Key Laboratory of Systems Biology, Shanghai Institutes for Biological Sciences, University of Chinese Academy of Sciences, Shanghai, China.

Received: 6 July 2014 Accepted: 8 January 2015

Published online: 27 January 2015

\section{References}

1. Adams LA, Angulo P, Lindor KD. Nonalcoholic fatty liver disease. CMAJ. 2005;172:899-905.

2. Cohen JC, Horton JD, Hobbs HH. Human fatty liver disease: old questions and new insights. Science. 2011;332:1519-23.

3. Williams KH, Shackel NA, Gorrell MD, McLennan SV, Twigg SM. Diabetes and nonalcoholic fatty liver disease: a pathogenic duo. Endocr Rev. 2013;34:84-129.

4. Armstrong MJ, Houlihan DD, Rowe IA. Pioglitazone, vitamin E, or placebo for nonalcoholic steatohepatitis. N Engl J Med. 2010;363:1185-6.

5. Lavine JE, Schwimmer JB, Van Natta ML, Molleston JP, Murray KF, Rosenthal $P$, et al. Effect of vitamin E or metformin for treatment of nonalcoholic fatty liver disease in children and adolescents: the TONIC randomized controlled trial. JAMA. 2011;305:1659-68.

6. Zhang J, Cao H, Zhang B, Cao H, Xu X, Ruan H, et al. Berberine potently attenuates intestinal polyps growth in ApcMin mice and familial adenomatous polyposis patients through inhibition of Wnt signalling. J Cell Mol Med. 2013;17:1484-93.

7. Z Zhang H, Wei J, Xue R, Wu JD, Zhao W, Wang ZZ, et al. Berberine lowers blood glucose in type 2 diabetes mellitus patients through increasing insulin receptor expression. Metabolism. 2010:59:285-92.

8. Zhou L, Wang X, Yang Y, Wu L, Li F, Zhang R, et al. Berberine attenuates CAMP-induced lipolysis via reducing the inhibition of phosphodiesterase in 3T3-L1 adipocytes. Biochim Biophys Acta. 2011;1812:527-35.

9. Kong W, Wei J, Abidi P, Lin M, Inaba S, Li C, et al. Berberine is a novel cholesterol-lowering drug working through a unique mechanism distinct from statins. Nat Med. 2004;10:1344-51.

10. Brusq JM, Ancellin N, Grondin P, Guillard R, Martin S, Saintillan Y, et al. Inhibition of lipid synthesis through activation of AMP kinase: an additional mechanism for the hypolipidemic effects of berberine. J Lipid Res. 2006;47:1281-8.

11. Kim WS, Lee YS, Cha SH, Jeong HW, Choe SS, Lee MR, et al. Berberine improves lipid dysregulation in obesity by controlling central and peripheral AMPK activity. Am J Physiol Endocrinol Metab. 2009;296:E812-9.

12. Chang $X$, Yan H, Fei J, Jiang M, Zhu H, Lu D, et al. Berberine reduces methylation of the MTTP promoter and alleviates fatty liver induced by a high-fat diet in rats. J Lipid Res. 2010;51:2504-15

13. Ge Y, Zhang Y, Li R, Chen W, Li Y, Chen G. Berberine regulated Gck, G6pc, Pck1 and Srebp-1c expression and activated AMP-activated protein kinase in primary rat hepatocytes. Int J Biol Sci. 2011;7:673-84.

14. Zhao HL, Sui Y, Qiao CF, Yip KY, Leung RK, Tsui SK, et al. Sustained antidiabetic effects of a berberine-containing Chinese herbal medicine through regulation of hepatic gene expression. Diabetes. 2012;61:933-43.

15. Cheng Z, Pang T, Gu M, Gao AH, Xie CM, Li JY, et al. Berberine-stimulated glucose uptake in L6 myotubes involves both AMPK and p38 MAPK. Biochim Biophys Acta. 2006;1760:1682-9.

16. Lee YS, Kim WS, Kim KH, Yoon MJ, Cho HJ, Shen Y, et al. Berberine, a natural plant product, activates AMP-activated protein kinase with beneficial 
metabolic effects in diabetic and insulin-resistant states. Diabetes. 2006;55:2256-64

17. Turner N, Li JY, Gosby A, To SW, Cheng Z, Miyoshi H, et al. Berberine and its more biologically available derivative, dihydroberberine, inhibit mitochondrial respiratory complex I: a mechanism for the action of berberine to activate AMP-activated protein kinase and improve insulin action. Diabetes. 2008;57:1414-8.

18. Hotamisligil GS. Endoplasmic reticulum stress and the inflammatory basis of metabolic disease. Cell. 2010;140:900-17.

19. Zhang $X$, Zhao Y, Zhang M, Pang $X, X u$ J, Kang C, et al. Structural changes of gut microbiota during berberine-mediated prevention of obesity and insulin resistance in high-fat diet-fed rats. PLoS One. 2012;7:e42529.

20. Wilusz JE, Sunwoo H, Spector DL. Long noncoding RNAs: functional surprises from the RNA world. Genes Dev. 2009;23:1494-504.

21. Mercer TR, Dinger ME, Mattick JS. Long non-coding RNAs: insights into functions. Nat Rev Genet. 2009;10:155-9.

22. Ponting $\mathrm{CP}$, Oliver PL, Reik W. Evolution and functions of long noncoding RNAs. Cell. 2009;136:629-41.

23. Wang KC, Chang HY. Molecular mechanisms of long noncoding RNAs. Mol Cell. 2011;43:904-14.

24. Pauli A, Rinn JL, Schier AF. Non-coding RNAs as regulators of embryogenesis. Nat Rev Genet. 2011;12:136-49.

25. Wapinski O, Chang HY. Long noncoding RNAs and human disease. Trends Cell Biol. 2011;21:354-61.

26. Folch J, Lees M, Sloane Stanley GH. A simple method for the isolation and purification of total lipides from animal tissues. J Biol Chem. 1957;226:497-509.

27. Livak KJ, Schmittgen TD. Analysis of relative gene expression data using real-time quantitative PCR and the 2(-Delta Delta C(T)) method. Methods. 2001:25:402-8.

28. Smyth GK, Michaud J, Scott HS. Use of within-array replicate spots for assessing differential expression in microarray experiments. Bioinformatics. 2005;21:2067-75

29. Huang DW, Sherman BT, Lempicki RA. Systematic and integrative analysis of large gene lists using DAVID bioinformatics resources. Nat Protoc. 2009;4:44-57.

30. Ashburner M, Ball CA, Blake JA, Botstein D, Butler H, Cherry JM, et al. Gene ontology: tool for the unification of biology. the gene ontology consortium. Nat Genet. 2000:25:25-9.

31. Altermann E, Klaenhammer TR. Pathway voyager: pathway mapping using the Kyoto Encyclopedia of Genes and Genomes (KEGG) database. BMC Genomics. 2005;6:60.

32. Langfelder P, Horvath S. WGCNA: an R package for weighted correlation network analysis. BMC Bioinformatics. 2008;9:559.

33. Saito R, Smoot ME, Ono K, Ruscheinski J, Wang PL, Lotia S, et al. A travel guide to Cytoscape plugins. Nat Methods. 2012;9:1069-76.

34. Lin MF, Jungreis I, Kellis M. PhyloCSF: a comparative genomics method to distinguish protein coding and non-coding regions. Bioinformatics. 2011;27: i275-82.

35. Agostini F, Zanzoni A, Klus P, Marchese D, Cirillo D, Tartaglia GG. catRAPID omics: a web server for large-scale prediction of protein-RNA interactions. Bioinformatics. 2013;29:2928-30

36. Xing LJ, Zhang L, Liu T, Hua YQ, Zheng PY, Ji G. Berberine reducing insulin resistance by up-regulating IRS-2 mRNA expression in nonalcoholic fatty liver disease (NAFLD) rat liver. Eur J Pharmacol. 2011;668:467-71.

37. Teodoro JS, Duarte FV, Gomes AP, Varela AT, Peixoto FM, Rolo AP, et al. Berberine reverts hepatic mitochondrial dysfunction in high-fat fed rats: a possible role for SirT3 activation. Mitochondrion. 2013;13:637-46.

38. Cullinan SB, Diehl JA. PERK-dependent activation of Nrf2 contributes to redox homeostasis and cell survival following endoplasmic reticulum stress. J Biol Chem. 2004;279:20108-17.

39. Sutherland AP, Zhang H, Zhang Y, Michaud M, Xie Z, Patti ME, et al. Zinc finger protein Zbtb20 is essential for postnatal survival and glucose homeostasis. Mol Cell Biol. 2009;29:2804-15.

40. Sun L, Goff LA, Trapnell C, Alexander R, Lo KA, Hacisuleyman E, et al. Long noncoding RNAs regulate adipogenesis. Proc Natl Acad Sci U S A. 2013:110:3387-92

41. Guttman M, Rinn JL. Modular regulatory principles of large non-coding RNAs. Nature. 2012:482:339-46.

42. DeNicola GM, Karreth FA, Humpton TJ, Gopinathan A, Wei C, Frese K, et al. Oncogene-induced Nrf2 transcription promotes ROS detoxification and tumorigenesis. Nature. 2011;475:106-9.
43. Xu J, Kulkarni SR, Donepudi AC, More VR, Slitt AL. Enhanced Nrf2 activity worsens insulin resistance, impairs lipid accumulation in adipose tissue, and increases hepatic steatosis in leptin-deficient mice. Diabetes. 2012;61:3208-18.

44. Okada K, Warabi E, Sugimoto H, Horie M, Gotoh N, Tokushige K, et al. Deletion of Nrf2 leads to rapid progression of steatohepatitis in mice fed atherogenic plus high-fat diet. J Gastroenterol. 2013:48:620-32.

45. Wang D, Wei Y, Pagliassotti MJ. Saturated fatty acids promote endoplasmic reticulum stress and liver injury in rats with hepatic steatosis. Endocrinology. 2006;147:943-51

46. Puri P, Mirshahi F, Cheung O, Natarajan R, Maher JW, Kellum JM, et al. Activation and dysregulation of the unfolded protein response in nonalcoholic fatty liver disease. Gastroenterology. 2008;134:568-76.

\section{Submit your next manuscript to BioMed Central and take full advantage of:}

- Convenient online submission

- Thorough peer review

- No space constraints or color figure charges

- Immediate publication on acceptance

- Inclusion in PubMed, CAS, Scopus and Google Scholar

- Research which is freely available for redistribution

Submit your manuscript at www.biomedcentral.com/submit
C) BioMed Central 